Université กำ
de Montréal

Serge Sultan, Université de Montréal

How to cite this article:

Ogez, D., Aramideh, J., Mizrahi, T., Charest, M.-C., Plante, C., Duval, M., \& Sultan, S. (2020). Does practising hypnosis-derived communication techniques by oncology nurses translate into reduced pain and distress in their patients? An exploratory study. British Journal of Pain.

https://doi.org/10.1177/2049463720932949

DOI: $\quad$ https://doi.org/10.1177/2049463720932949

PMID: in process 
Does practising hypnosis-derived communication techniques by oncology nurses translate into reduced pain and distress in their patients? An exploratory study

David Ogez ${ }^{1,2}$, Jennifer Aramideh ${ }^{1,2}$, Terry Mizrahi ${ }^{1}$, Marie-Claude Charest ${ }^{1}$, Caroline

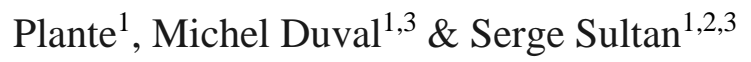

${ }^{1}$ Department of Hematology-Oncology, CHU Sainte-Justine, Montréal, Québec, Canada

${ }^{2}$ Department of Psychology, Université de Montréal, Montréal, Québec, Canada

${ }^{3}$ Department of Pediatrics, Université de Montréal, Montréal, Québec, Canada

Keywords: Procedural pain, distress, pediatric, hematology oncology, Hypnotic communication, nurses' training 
Does practising hypnosis-derived communication techniques by oncology nurses translate into reduced pain and distress in their patients? An exploratory study

Abstract: Objectives: To explore the effects of a hypnotic communication (HC) training for pediatric nurses in decreasing patients' pain and distress during venipunctures. Methods: A 4day theoretical and practical $\mathrm{HC}$ training was offered to 5 pediatric oncology nurses. The effects of $\mathrm{HC}$ were tested with 22 young cancer patients (13 girls, 9 boys, $10 \pm 4$ years) over 4 timepoints, with 88 encounters being video-recorded and coded in stable professional-patient dyads. Patients' pain and distress were rated by patients and parents with visual analogue scales and coded from recordings using the Faces, Legs, Activity, Cry and Consolability (FLACC) scale. Results: We observed a significant decrease in pre-post distress reported by parents $(d=0.45, \mathrm{p}=0.046)$. Two (out of 5$)$ nurses with higher skills acquisition had larger reduction in patients' self-reported pain $(d=1.03, \mathrm{p}=0.028)$, parents perceived pain $(d=1.09$, $\mathrm{p}=0.042)$, distress $(d=1.05, \mathrm{p}=0.043)$ as well as observed pain $(d=1.22 \mathrm{p}=0.025)$. Favorable results on pain and distress did not maintain at follow-up. Conclusion and clinical implications: Training nurses in HC may translate into improved pain and distress in patients, both self-rated and observed provided that skills are used in practice. $\mathrm{HC}$ training is a promising non-pharmacological intervention to address pain in pediatrics.

Keywords: Procedural pain, distress, pediatric, hematology oncology, Hypnotic communication, nurses' training 


\section{Does practising hypnosis-derived communication techniques by oncology nurses translate into reduced pain and distress in their patients? An exploratory study}

\section{$\underline{\text { Introduction }}$}

Children with cancer experience repetitive painful needle procedures along the trajectory. ${ }^{1}$ Previous studies have observed that medical procedures can cause significant medical stress and may complicate care, yield avoidance or adherence issues. ${ }^{2}$ It is therefore crucial to manage pain and distress from an early age. Pharmacological interventions such as local anesthetic (EMLA cream $\left.\AA^{3}\right)$, as well as non-pharmacological (e.g. Buzzy ${ }^{4}{ }^{4}$, distraction ${ }^{5}$, hypnosis ${ }^{1,6}$ ) are typically used by the healthcare team to prevent and manage procedural pain.

Among non-pharmacological interventions, hypnosis has been considered as highly relevant due to the high suggestibility of children. ${ }^{7}$ Previous studies have demonstrated the efficacy of hypnosis as a single or combined intervention, in reducing procedural pain ${ }^{7-17}$ and distress $^{7,8,11-15}$ in children with cancer. In these studies, healthcare professionals performed the medical procedure while another professional (e.g. hypnotherapist) provided the hypnosis talk. This setting is a major barrier to the use of these techniques in the day-to-day pediatric clinics. ${ }^{18}$ Recently, nurses have themselves used the hypnotic communication (HC) skills while performing the painful procedure. ${ }^{19}$ To date such studies are extremely rare and have not linked the professional's behaviour with patient's level of pain/distress.

Mizrahi et al. developed and evaluated a short training course in $\mathrm{HC}$ for nurses in pediatric hematology-oncology. ${ }^{19}$ A hypnosis-certified psychologist conducted four 5-hour sessions of $\mathrm{HC}$ training (Figure 1). The content was adapted from manuals of hypnotic intervention in children and is available in previous reports. ${ }^{19}, 20$ When studying skills acquisition in nurses, authors found medium-large desirable behavioral changes following the training in a group of nurses. ${ }^{21}$ In the present study, we aim at reporting changes at the patients' 
level. First, we assessed whether training in hypnotic communication was associated with expected improvements in pain and distress. Second, we explored whether such improvements would be related with the use of $\mathrm{HC}$ techniques in practice.

Figure 1: Content of the hypnotic communication training sessions

\section{Methods}

This exploratory study was conducted at the Hematology-Oncology daycare clinic at a large tertiary Canadian hospital. Ethical approval was received by our local Research Ethics Committee (\#2015-849) and all participants (patients and nurses) provided written informed consent. The reporting of this study was previously published and is compliant with the TREND Statement. ${ }^{19,22}$

\section{Inclusion and exclusion Criteria}

To be included in this study, cancer patients had to be aged between 5 and 18 years old, have regular follow-up at the clinic and understand French. Patients who had previously been exposed to hypnosis or hypnosis-derived communication were excluded, as were those who came to the clinic for an unexpected appointment or those with a psychiatric disorder. Nurses were required to work at the outpatient hematology-oncology daycare clinic, have previous experience performing venipuncture in pediatrics and have no prior experience in hypnosis or hypnosis-derived communication.

\section{Participants and setting}

We invited 6 nurses and 36 patients to participate in this study. Nurses took part in the 4-day HC training at our hospital. Using a convenience sampling method, the research assistant 
assigned 6 patients from the clinic's computer database to each nurse. The first six patients meeting the criteria were contacted by phone and received preliminary information on the study. Patients and their parent(s) subsequently met with a researcher (TM or JA) to obtain additional information about the study and sign their consent. Then, patients were received at the daycare hematology-oncology clinic by the nurses for a venipuncture (VP), which was video-recorded. After the VP, patients, parents and the researcher completed evaluation scales.

Patients' distress and pain, and nurses' HC skills were assessed at 4 time-points: 2 in pre-training (T1 and T2 on average respectively 119 and 148 days before the training) and 2 in post-training (T3 and T4 on average respectively 137 and 203 days after the training). The training took place on 16, 17, 23 and 24 September 2015 and data collection was carried out from March 2015 to November 2016.

\section{Measures}

Socio-demographic and medical information: For nurses, we collected age and years of service. For patients, we collected age, sex, cancer diagnosis and the date of diagnosis.

Self-reported pain and distress: Visual analogic scales (VAS) were used in this study to assess fear and pain of venipuncture rated by children and their parents. The VAS is a wellvalidated, appropriate, efficient and easy to use scale in children from 5 to 18 years old. ${ }^{23-25}$ As the concept of distress is not expected to be mastered by the younger children of this sample, we used self-report with anchor term referring to levels of fear. ${ }^{26}$ Respondents were asked "how much you/your child feared the venipuncture", from 0 to 10 , "no fear at all" to "a lot of fear", respectively. To assess pain (children) and perception of pain (parents), respondents replied to the item "how much pain did you/your child feel during the venipuncture?", 0 being "no pain at all", and 10 "extreme pain". 
Observed pain: We used the FLACC (Faces, Legs, Activity, Cry and Consolability) Scale to code pain from video recorded encounters. ${ }^{27-29}$ This internationally validated scale measures procedural pain and distress in children from birth to 18 years. Research has identified substantial levels of pain and distress in young children using FLACC during commonly performed emergency department procedures. ${ }^{19}$ Inter-rater reliability of FLACC in this dataset was excellent (median ICC $=0.789$ ).

Sainte-Justine Hypnotic Communication Assessment Scale (SJ-HCAS): This scale was used to assess communication behaviours in video-recorded nurse-patient encounters. ${ }^{20}$ It is based on 11 core items of hypnosis-derived communication to prevent pain and distress in children. For each item, an independent rater evaluates whether the behavior is present (1) or absent (0). Two count scores are computed, one on the quality of the relationship (subscore 0 to 5) and one on the quality of the communication technique (subscore 0 to 6 ). A total score is computed by adding all 11 items (range $0-11$ ). The score is transformed to a percentage. In this dataset, we had excellent inter-rater reliability for the total score and the two subscores (median ICC $=0.879$ ), when raters were blind of assessment time-points and when raters had different

professional backgrounds. ${ }^{20}$ At the same time, nurses were asked to answer a question related to the technique used: 0 = "no technique", 1 = "guided imagery", $2=$ "switch", $3=$ "pain transformation".

\section{Data Analysis}

To explore stability between the two pre-training time points, we computed differences (Cohen's d) and absolute stability ${ }^{30}$ on the VAS measures and the FLACC. For comparisons of pain and distress over time, we used the non-parametric Wilcoxon test. Effect sizes (Cohen's d) were calculated to compare the differences between pre-training and post-training assessment for the whole sample and for each nurse in particular. An alpha level of 0.05 was set for 
inference statistics. As the sample size was limited, and consistent with the exploratory nature of the study, we put more emphasis on effect sizes. Analyses were performed with the SPSS 25 software.

\section{$\underline{\text { Results }}$}

\section{Final sample and preliminary analyses}

We included 6 female pediatric oncology nurses aged $33 \pm 6$ years, and 33 of their cancer patients (16 boys, 17 girls) aged $10 \pm 4$ years. Among these participants, one nurse stopped training for maternity leave, and 7 patients did not complete the study: 2 passed away and 5 dropped out (see figure 2). Five nurses aged $33 \pm 5$ years completed the full $\mathrm{CH}$ training. Twenty-two patients (13 girls, 9 boys) aged 10 14 years participated in all time-points with the same nurse and were available for complete pre-, post-, and follow-up analyses. Data derive from 88 stable dyad nurse-patient encounters.

When considering data points before training, we observed stability in all measures: SJHCAS scores $(\mathrm{ICCs}=0.630-0.766, d=0.141-0.167)$, VAS scales $(\mathrm{ICCs}=0.681-0.862, d=-0.216$ 0.000 ) and the FLACC score (ICCs $=-0.058, d=-0.058$ ). Consequently, we averaged both pretraining time points to form one baseline value for each outcome.

Figure 2. Flow chart

\section{Changes in pain and distress following the training}

When considering all available nurse-patient interactions $(n=88)$, no significant change on pain or distress reported by patient, pain reported by parent, and observed pain was detected, either in post-training or follow-up $(d s=0.26-0.32)$. We found a significant reduction in post- 
training distress scores as perceived by parents $(\mathrm{Z}=-1.993, \mathrm{p}=0.046, d=0.45$, small $)$, not maintained in follow-up.

However, when investigating the same results across nurses, we found heterogeneity in the sample, with significant changes for nurses C and D. For these nurses, pre-post comparisons showed large differences on self-reported pain $(d s=0.94-1.03)$, parent-reported pain $(d s=0.90$ 1.19), observed pain ( $d s=0.95-1.43)$, and medium-large differences on parent-reported distress $(d s=0.73-1.82)$. We did not observe changes on pre-post self-reported distress $(d s=0.23-0.28)$ and the maintenance of these effects in follow-up. In contrast, in nurses A, B, F, we observed negligible changes, with the exception of patient 'self-report distress pre-post in Nurse A $(d=0.84)$ and Observed pain pre-follow up in Nurse F $(d=0.96)$ (Supplementary material Figure S1).

\section{Role of HC skills acquisition and technique use}

The observed changes in effect sizes among these nurses were consistent with the results demonstrated in the skills acquisition study, showing larger scores of hypnotic communication techniques among nurses $\mathrm{C}$ and $\mathrm{D}(d s=1.21-1.51)$, as compared to $\mathrm{A}, \mathrm{B}$ and $\mathrm{F}(d s=0.32-0.71) .{ }^{21}$ When comparing changes at the patients level for the two groups of nurses $[C, D]$ vs $[A, B, F]$, we found a large reduction in pain self-reported $(\mathrm{Z}=-2.201, \mathrm{p}=0.028, d=1.03)$, and parentreported $(\mathrm{Z}=-2.032, \mathrm{p}=0.042, d=1.09)$, observed pain $(\mathrm{Z}=-2.243, \mathrm{p}=0.025, d=1.22)$ and parentreported distress $(\mathrm{Z}=-2.023, \mathrm{p}=0.043, d=1.05)$ for $[\mathrm{C}, \mathrm{D}]$, while no significant results were observed for the other group $[\mathrm{A}, \mathrm{B}, \mathrm{F}]$. In summary, nurses who improved most on their communication skills also demonstrated greater changes on pain and distress (Figure 3). Yet, effects were limited to post-training and did not maintain over time.

To further document the relationship between the use of techniques with the observed outcomes at the patient level, we focused on video-recorded interactions and compared the 
observed pain (FLACC scores) in encounters demonstrating desired communication techniques (proportions of encounters post: $n=9,42 \%$, follow-up: $n=8,40 \%$ ) vs encounters with no such techniques. When selecting encounters demonstrating the use of $\mathrm{HC}$ techniques, we found a maintained reduction in observed pain in the former group (Pre-post: $Z=-2.255, p=0.024$, $d=1.05$; pre-follow-up: $\mathrm{Z}=-2.028, \mathrm{p}=0.043, d=0.93$ ). This was not observed when techniques were not used (pre-post: $\mathrm{Z}=-1.350, \mathrm{p}=0.177, d=-0.41$; pre-follow-up: $\mathrm{Z}=-1.548, \mathrm{p}=0.122, d=-$ 0.43). Notably, only the "guided imagery" technique was used by nurses.

Figure 3. Cohen's d across time-points for patients' pain and distress scores for nurses C \& D vs nurses A, B \& F

\section{$\underline{\text { Discussion }}$}

The management of procedural pain requires the evaluation of simple and easily applicable techniques in healthcare networks. ${ }^{2,31}$ The present project aimed to meet this need by exploring patient-level effects of a hypnotic communication training for pediatric nurses. ${ }^{19}$ Following training, we found a reduction on self-reported pain, parent-reported pain, parentreported distress and observed pain, but only when nurses showed high skills acquisition.

While our first objective was to evaluate whether training in hypnotic communication was associated with improvements in pain and distress, we found little support for this when considering the whole group. Yet, there was heterogeneity among the participating nurses, and when isolating those who showed higher skills acquisition, we observed favorable short-time reduction in self-reported pain, parent-reported pain, observed pain and parent-reported distress. This is coherent with the model of the training and is an encouragement to use and refine this type of training. Yet, changes did not hold overtime at the patient level in those who improved their skills in HC. Although disappointing, this result is consistent with the Nurse Competency Acquisition Study. ${ }^{21}$ In this study, after completing the training, the results showed a decrease in nurses' HC skills. This suggest that skills should be reinforced to maintain benefits 
in the long term. To address this limitation, continuing education and supervision will probably be important. As it is observed in medical pedagogy, follow-up training is often required to enhance health caregivers' skills. ${ }^{32}$ One important output of this research points to a refinement of the training in adding supervision, or reorganizing future training over time so that sessions may better benefit from application to practice (e.g. four weekly sessions instead of 4 consecutive days). Additional refinements were also suggested by nurses in a previous qualitative survey. ${ }^{21}$

Interestingly, when focusing on encounters where nurses actually used the communication techniques, we found improvements in observed pain, both post-training and at follow-up. This means that patients who encountered a nurse who used the HC technique experienced a significant reduction in observed video-coded pain, whereas this was not the case when the nurse did not practice HC. It is the first time that nurses hypnotic behavior is directly associated with pain reduction, and strongly suggests that it would be beneficial to use adequate $\mathrm{HC}$ techniques to prevent pain in children. Importantly, this result was evaluated by tracking stable dyads over time, and thereby controlling for several interpersonal biases. Although we know that hypnotic techniques are very effective in reducing pain according to the literature, previous studies demonstrating the positive effects of $\mathrm{HC}$ have been evaluated with two separate providers: the nurse and a hypnotherapist. ${ }^{10-15}$ In the present study, we observed selfinitiated HC effects. This highlights the potential impact of an inexpensive and easy-toimplement non-pharmacological intervention on pain and procedural distress.

Despite these encouraging results, we must recognize the study limitations. Firstly, the samples were small and analyses lacked power. We tried to deal with this issue by emphasizing effect sizes in our interpretations, as it is usual in exploratory studies. The study should be replicated in a larger pilot design with a comparison to a control group, following recommendations for program development. ${ }^{33}$ Though, the results lend support to favorable 
outcomes when skills are used in practice. Secondly, we had a significant dropout rate, particularly due to the maternity leave of a nurse, which caused the exclusion of several patients from the study. This important feasibility aspect should be anticipated in future trials aiming at testing the effects of HC training. Finally, we were unable to control the time laps between encounters due to the clinical work organization. To limit time bias, it would be important in future trials to better standardize time points. As this appeared difficult to do in practice when stable dyads were observed, one could observe nurses' skills in controlled simulation protocols. In conclusion, this exploratory study with 22 stable dyads followed over time showed that a hypnotic communication training of nurses was associated with a short-time reduction in procedural pain and distress of patients in nurses who acquired skills following training. Notably, there was a more durable change in observed pain in encounters where the nurse effectively used the $\mathrm{HC}$ communication techniques. This supports further developments of simple HC training to meet the increasing demand for non-pharmacological, low-cost and easyto-implement techniques in pediatric oncology. Future research should refine HC training and further explore the link between $\mathrm{HC}$ communication behaviors in professionals and the pain response in pediatric patients. 


\section{$\underline{\text { References }}$}

1. Birnie KA, Noel M, Parker JA, et al. Systematic Review and Meta-Analysis of Distraction and Hypnosis for Needle-Related Pain and Distress in Children and Adolescents. Journal of Pediatric Psychology 2014; 39: 783-808. DOI: 10.1093/jpepsy/jsu029.

2. Pai AL and Kazak AE. Pediatric medical traumatic stress in pediatric oncology: family systems interventions. Current Opinion in Pediatrics 2006; 18: 558-562. 2006/09/14. DOI: 10.1097/01.mop.0000245358.06326.e9.

3. Rogers TL and Ostrow CL. The use of EMLA cream to decrease venipuncture pain in children. J Pediatr Nurs 2004; 19: 33-39. 2004/02/14. DOI: 10.1016/j.pedn.2003.09.005.

4. Şahiner NC, İnal S and Akbay AS. The effect of combined stimulation of external cold and vibration during immunization on pain and anxiety levels in children. Journal of PeriAnesthesia Nursing 2015; 30: 228-235.

5. $\quad$ Koller D and Goldman RD. Distraction Techniques for Children Undergoing Procedures: A Critical Review of Pediatric Research. Journal of Pediatric Nursing 2012; 27: 652-681. DOI: https://doi.org/10.1016/j.pedn.2011.08.001.

6. Accardi MC and Milling LS. The effectiveness of hypnosis for reducing procedurerelated pain in children and adolescents: a comprehensive methodological review. Journal of behavioral medicine 2009; 32: 328-339. 2009/03/04. DOI: 10.1007/s10865009-9207-6.

7. Kuttner L, Bowman M and Teasdale M. Psychological Treatment of Distress, Pain, and Anxiety for Young Children with Cancer. Journal of Developmental and Behavioral Pediatrics 1988; 9: 374-382.

8. Hawkins PJ, Liossi C, Ewart BW, et al. Hypnosis in the alleviation of procedure related pain and distress in paediatric oncology patients. Contemporary Hypnosis 1998; 15: 199-207. DOI: 10.1002/ch.135.

9. Hilgard JR and Lebaron S. Relief of anxiety and pain in children and adolescents with cancer: Quantitative measures and clinical observations. International Journal of Clinical and Experimental Hypnosis 1982; 30: 417-442. DOI:

10.1080/00207148208407277.

10. Katz ER, Kellerman J and Ellenberg L. Hypnosis in the reduction of acute pain and distress in children with cancer. Journal of Pediatric Psychology 1987; 12: 379-394.

11. Liossi $\mathrm{C}$ and Hatira P. Clinical hypnosis versus cognitive behavioral training for pain management with pediatric cancer patients undergoing bone marrow aspirations. International Journal of Clinical and Experimental Hypnosis 1999; 47: 104-116. 1999/04/20. DOI: 10.1080/00207149908410025.

12. Liossi $\mathrm{C}$ and Hatira P. Clinical hypnosis in the alleviation of procedure-related pain in pediatric oncology patients. International Journal of Clinical and Experimental Hypnosis 2003; 51: 4-28. 2003/06/27. DOI: 10.1076/iceh.51.1.4.14064.

13. Liossi C, White P and Hatira P. Randomized clinical trial of local anesthetic versus a combination of local anesthetic with self-hypnosis in the management of pediatric procedure-related pain. Health Psychology 2006; 25: 307-315. 2006/05/25. DOI: 10.1037/0278-6133.25.3.307. 
14. Liossi C, White P and Hatira P. A randomized clinical trial of a brief hypnosis intervention to control venepuncture-related pain of paediatric cancer patients. Pain 2009; 142: 255-263. DOI: http://dx.doi.org/10.1016/j.pain.2009.01.017.

15. Smith JT, Barabasz A and Barabasz M. Comparison of hypnosis and distraction in severely ill children undergoing painful medical procedures. Journal of Counseling Psychology 1996; 43: 187-195. DOI: 10.1037/0022-0167.43.2.187.

16. Wall VJ and Womack W. Hypnotic versus Active Cognitive Strategies for Alleviation of Procedural Distress in Pediatric Oncology Patients. American Journal of Clinical Hypnosis 1989; 31: 181-191. DOI: 10.1080/00029157.1989.10402887.

17. Zeltzer L and LeBaron S. Hypnosis and nonhypnotic techniques for reduction of pain and anxiety during painful procedures in children and adolescents with cancer. The Journal of Pediatrics 1982; 101: 1032-1035. 1982/12/01.

18. Yeh VM, Schnur JB and Montgomery GH. Disseminating hypnosis to health care settings: Applying the RE-AIM framework. Psychology of Consciousness 2014; 1: 213-228. DOI: $10.1037 / \mathrm{cns} 0000012$.

19. Mizrahi T, Sultan S, Charest M-C, et al. Training nurses in basic hypno-analgesia techniques to reduce procedural distress and pain in children: A feasibility trial. Cogent Medicine 2016; 3. DOI: 10.1080/2331205x.2016.1165083.

20. Aramideh J, Mizrahi T, Charest M-C, et al. Development and inter-rater reliability of a tool assessing hypnotic communication behaviours adopted by nurses caring for children with cancer: The Sainte-Justine Hypnotic Communication Assessment Scale. Complementary Therapies in Medicine 2018; 37: 178-184. DOI:

https://doi.org/10.1016/i.ctim.2017.11.013.

21. Aramideh J, Ogez D, Mizrahi T, et al. Do professionals change their communication behaviours following a training in hypnosis-derived communication? A feasibility study in pediatric oncology.

22. Des Jarlais DC, Lyles C, Crepaz N, et al. Improving the Reporting Quality of Nonrandomized Evaluations of Behavioral and Public Health Interventions: The TREND Statement. American Journal of Public Health 2004; 94: 361-366.

23. Crandall M, Lammers $C$, Senders $C$, et al. Initial validation of a numeric zero to ten scale to measure children's state anxiety. Anesthesia and analgesia 2007; 105: 12501253, table of contents. DOI: 10.1213/01.ane.0000284700.59088.8b.

24. Babl FE, Mandrawa C, O'Sullivan R, et al. Procedural pain and distress in young children as perceived by medical and nursing staff. Paediatric anaesthesia 2008; 18 : 412-419. DOI: 10.1111/j.1460-9592.2008.02458.x.

25. Ortiz MI, Lopez-Zarco M and Arreola-Bautista EJ. Procedural pain and anxiety in paediatric patients in a Mexican emergency department. Journal of advanced nursing 2012; 68: 2700-2709. DOI: 10.1111/j.1365-2648.2012.05969.x.

26. Dufresne A, Dugas MA, Samson Y, et al. Do children undergoing cancer procedures under pharmacological sedation still report pain and anxiety? A preliminary study. Pain medicine 2010; 11: 215-223. DOI: 10.1111/j.1526-4637.2009.00701.x.

27. Willis MH, Merkel SI, Voepel-Lewis T, et al. FLACC Behavioral Pain Assessment Scale: a comparison with the child's self-report. Pediatric nursing 2003; 29: 195-198.

28. Nilsson S, Finnstrom B and Kokinsky E. The FLACC behavioral scale for procedural pain assessment in children aged 5-16 years. Paediatric anaesthesia 2008; 18: 767-774. DOI: 10.1111/j.1460-9592.2008.02655.x.

29. Blount RL and Loiselle KA. Behavioural assessment of pediatric pain. Pain research \& management : the journal of the Canadian Pain Society= journal de la societe canadienne pour le traitement de la douleur 2009; 14: 47-52. 
30. Cicchetti DV. Guidelines, criteria, and rules of thumb for evaluating normed and standardized assessment instruments in psychology. Psychological assessment 1994; 6: 284-290.

31. Po C, Benini F, Sainati L, et al. The management of procedural pain at the Italian Centers of Pediatric Hematology-Oncology: state-of-the-art and future directions. Supportive Care in Cancer 2012; 20: 2407-2414. 2012/01/03. DOI: 10.1007/s00520011-1347-x.

32. Snowdon DA, Leggat SG and Taylor NF. Does clinical supervision of healthcare professionals improve effectiveness of care and patient experience? A systematic review. BMC Health Serv Res 2017; 17: 786. 2017/12/01. DOI: 10.1186/s12913-0172739-5.

33. Czajkowski SM, Powell LH, Adler N, et al. From ideas to efficacy: The ORBIT model for developing behavioral treatments for chronic diseases. Health Psychol 2015; 34: 971-982. 2015/02/03. DOI: 10.1037/hea0000161. 
Figure 1. Content of the hypnotic communication techniques (HCTech) training sessions

\begin{tabular}{|c|c|c|c|}
\hline Session 1 & Session 2 & Session 3 & Session 4 \\
\hline $\begin{array}{l}\text { Themes presented } \\
\text { - Basics of clinical hypnosis } \\
\text { - Ericksonian and clinical } \\
\text { Hypnosis } \\
\text { - Hypnosis in healthcare } \\
\text { system } \\
\text { - Ethical challenges in } \\
\text { pediatrics } \\
\text { - Definition of pediatric pain } \\
\text { - Procedural pain and anxiety }\end{array}$ & $\begin{array}{l}\text { Themes presented } \\
\text { - Nurse-patient relationship } \\
\text { - Synchronisation technique } \\
\text { (pacing-leading) } \\
\text { - Language } \\
\text { - Distraction and hypnoanalgesia } \\
\text { - Hypnotic trance } \\
\text { - Hypnoanalgesia and post- } \\
\text { hypnotic suggestions } \\
\text { - Basic hypnoanalgesia concepts }\end{array}$ & $\begin{array}{l}\text { Practical exercises } \\
\text { - Role-play } \\
\text { - Feedback from the trainer } \\
\text { - Additional techniques: } \\
\text { "Switch", pain } \\
\text { transformation, bubble, } \\
\text { guided imagery } \\
\text { - Use of HCTech in the clinic } \\
\text { under the supervision of the } \\
\text { trainer }\end{array}$ & $\begin{array}{l}\text { Supervisions } \\
\text {-HCTech into practice } \\
\text { with patients under the } \\
\text { observation of the } \\
\text { trainer } \\
\text { - Feedback }\end{array}$ \\
\hline & $\begin{array}{l}\text { Practical exercises } \\
\text { Different techniques: Deep } \\
\text { breathing, the pleasant place, } \\
\text { sensation changes suggestions, } \\
\text { the magic glove }\end{array}$ & & \\
\hline
\end{tabular}


Figure 2. Flow chart
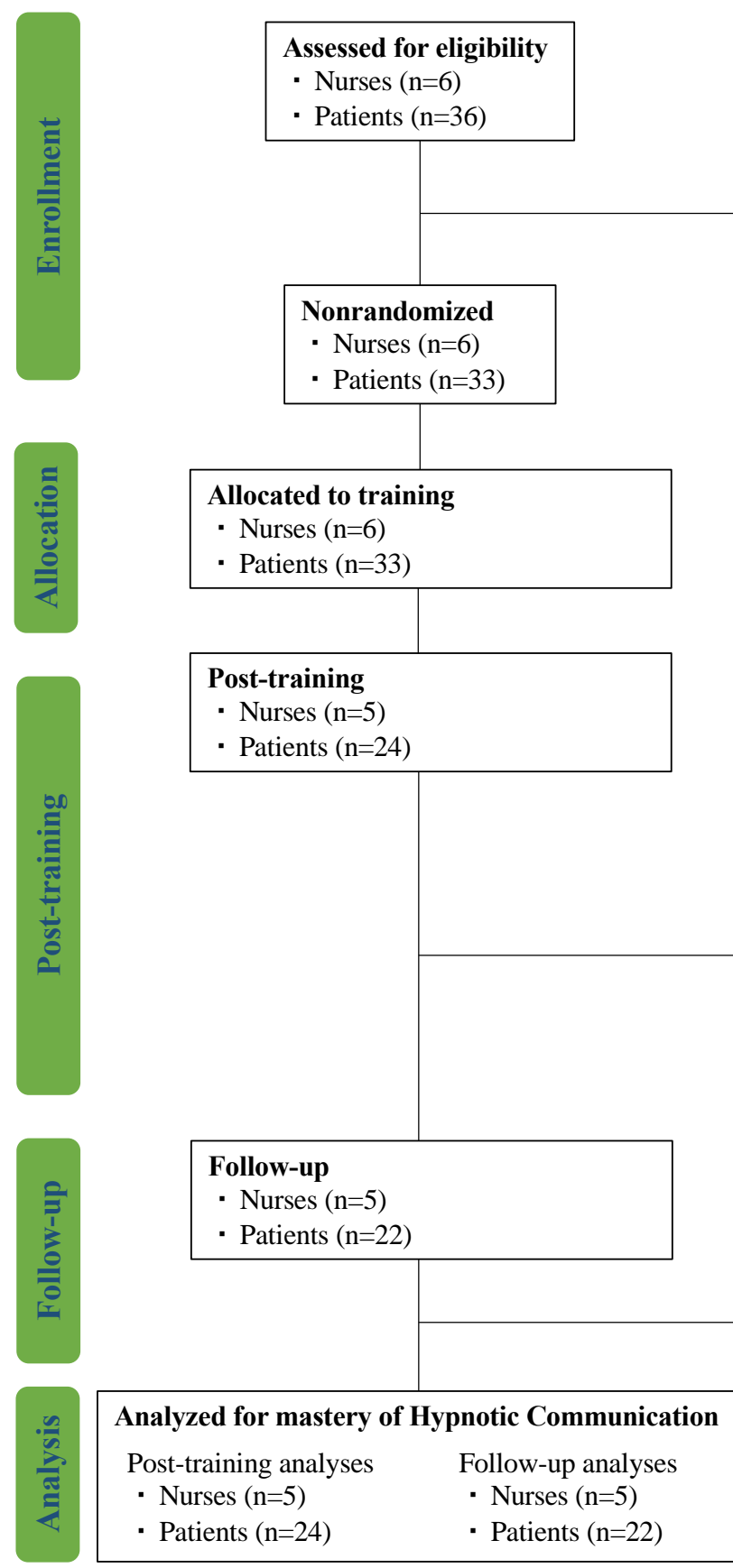

\section{Declined}

- Patients (n=3)

Not interested $(n=2)$

Does not want to be exposed to hypnosis $(n=1)$

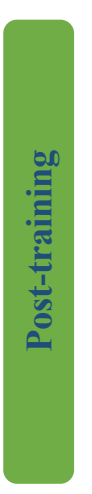

\section{Lost to post-training}

- Nurses ( $\mathrm{n}=1)$

Maternity leave $(\mathrm{n}=1)$

- Patients ( $\mathrm{n}=9)$

Deceased $(n=2)$

No longer wanted to undergo venipunctures $(n=1)$

No longer wanted to be filmed $(\mathrm{n}=1)$

No longer wanted to have the designated nurse perform the venipunctures $(n=1)$

Did not complete all 4 time-points with the same nurse due to their nurse's maternity leave $(n=4)$

\section{Lost to follow-up}

- Patients $(n=2)$

Unable to complete the final time-point $(n=2)$

Analyzed for mastery of Hypnotic Communication

Post-training analyses

- Nurses $(\mathrm{n}=5)$

- Patients $(n=24)$
Follow-up analyses

- Patients $(\mathrm{n}=22)$ 
Figure 3. Cohen's $d$ across time-points for patients' pain and distress scores for nurses $C$, D compared to nurses $A, B, F$

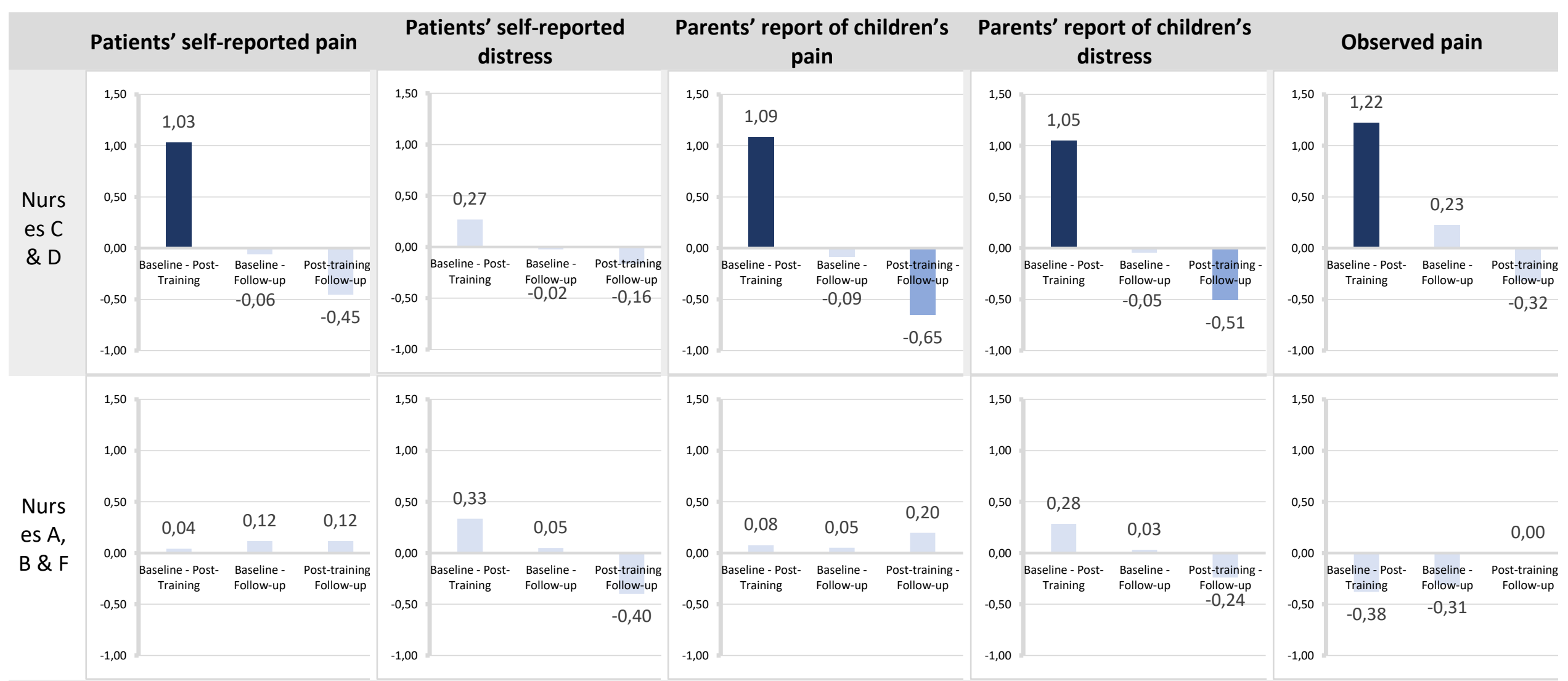

Note: Effect sizes $(d): \quad$ small $(0.20-0.50)$, medium $(0.50-0.80)$, and $\quad$ large $(0.80$ or higher)

Positives ds indicate a decrease in pain/distress 
Figure S1. Cohen's $d$ across time-points for patients' pain and distress scores across nurses

Patients' self-reported pain (0-10 VAS)

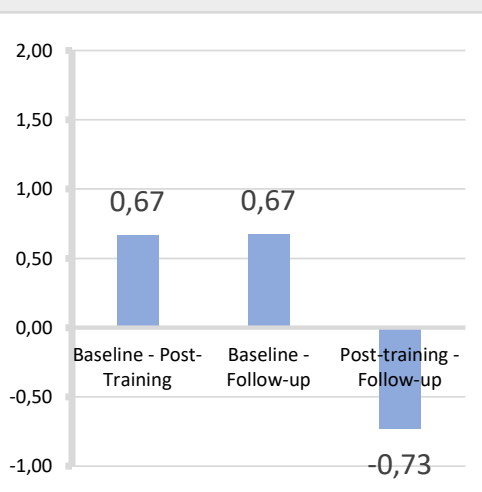

Patients' self-reported distress (0-10 VAS)

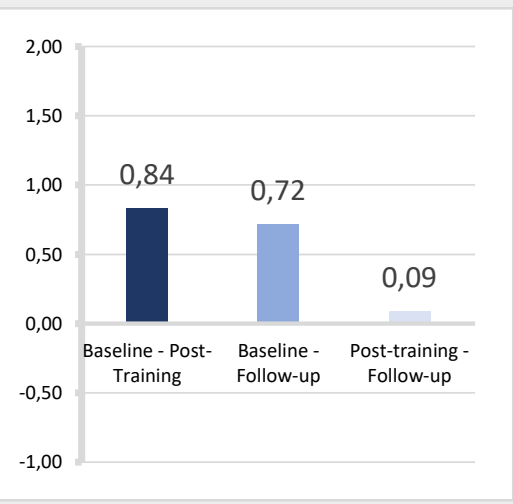

Parents' report of children's pain (0-10 VAS)

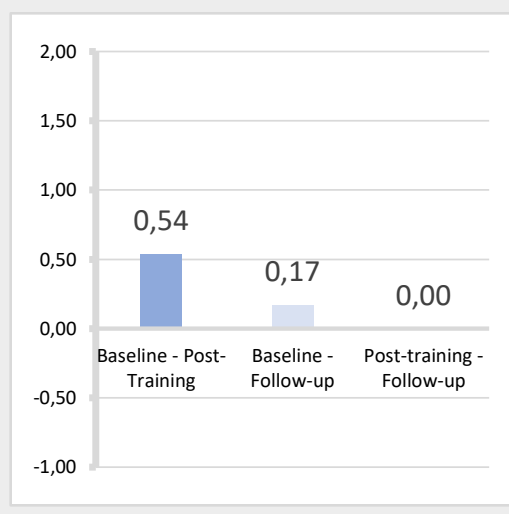

Parents' report of children's distress (0-10 VAS)

Observed pain (FLACC)

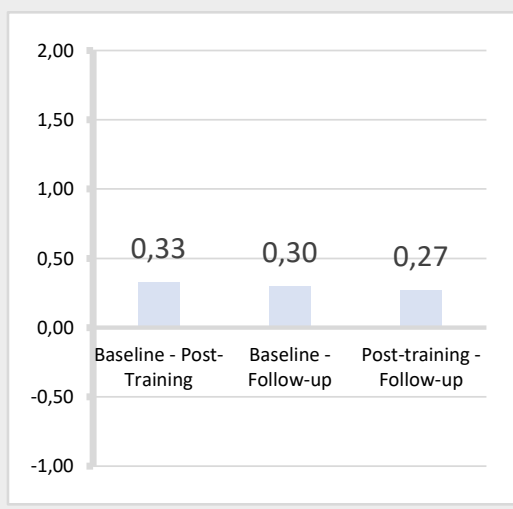

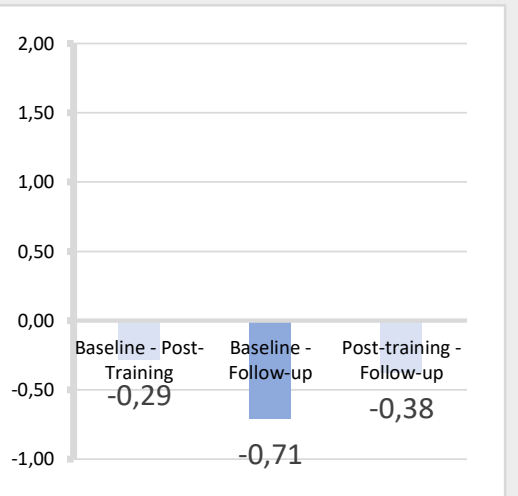

Nurse B
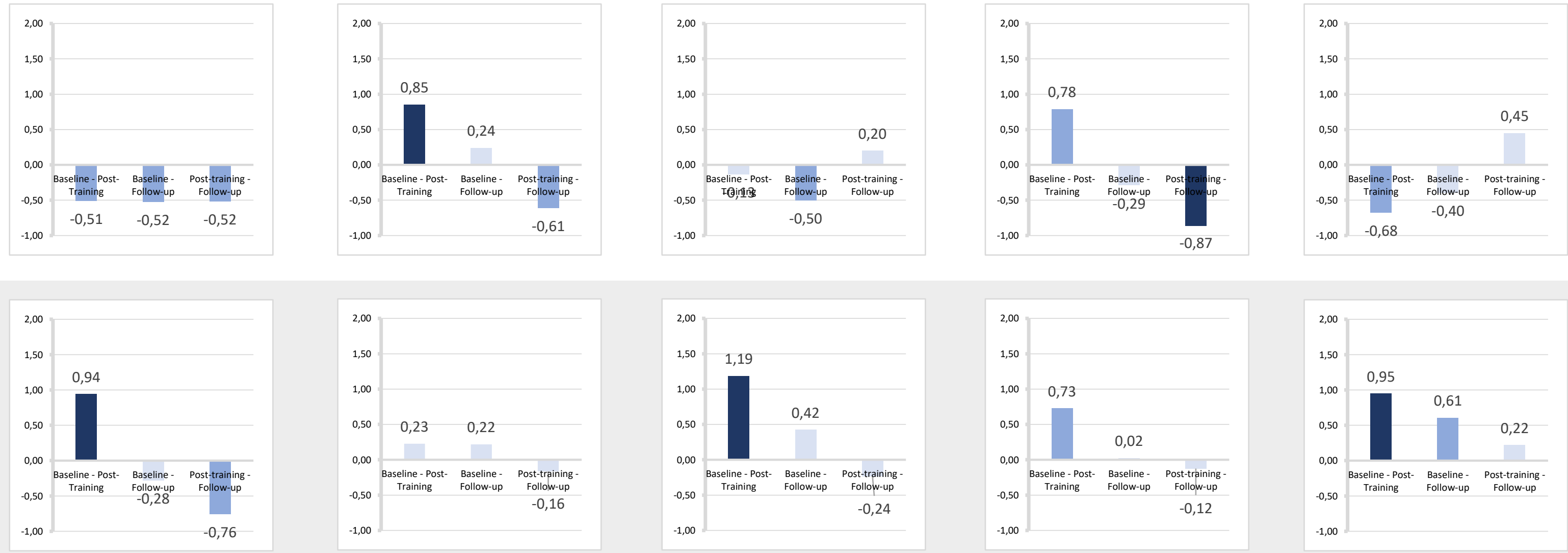
Nurse D
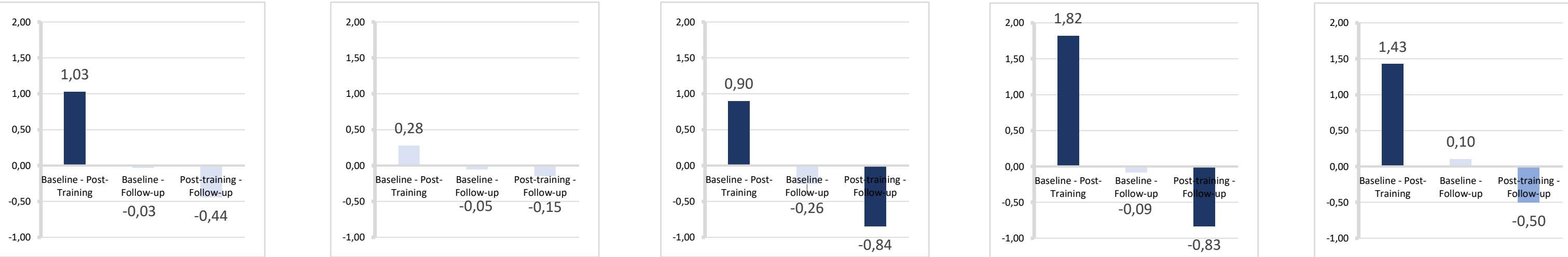

Nurse F

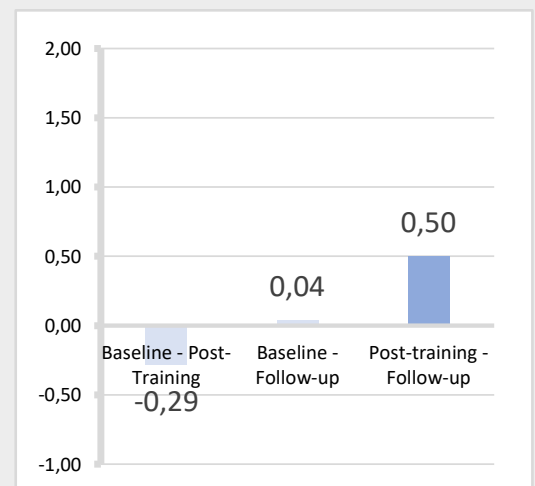

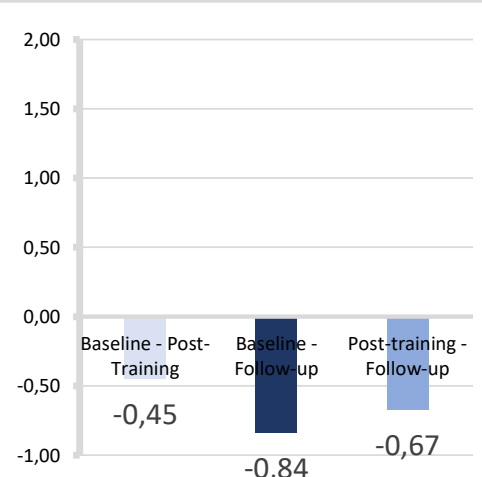

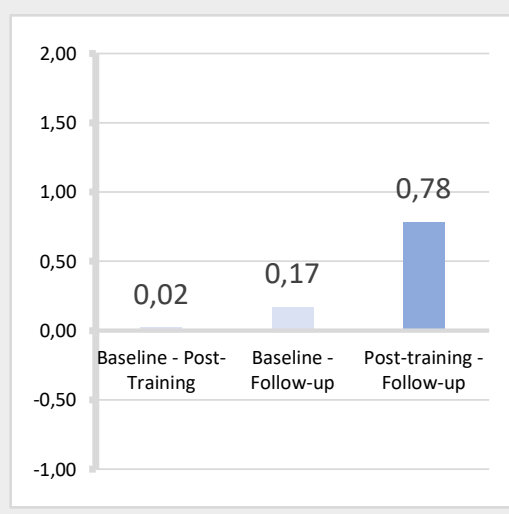

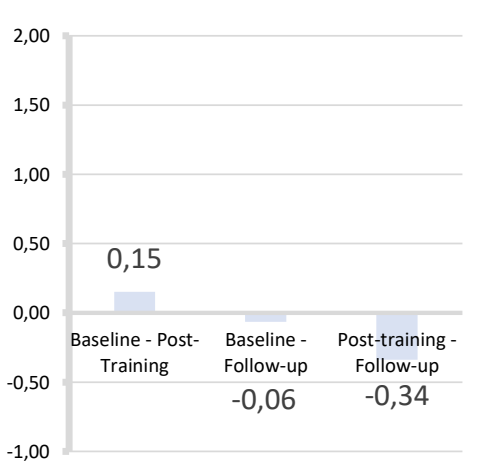

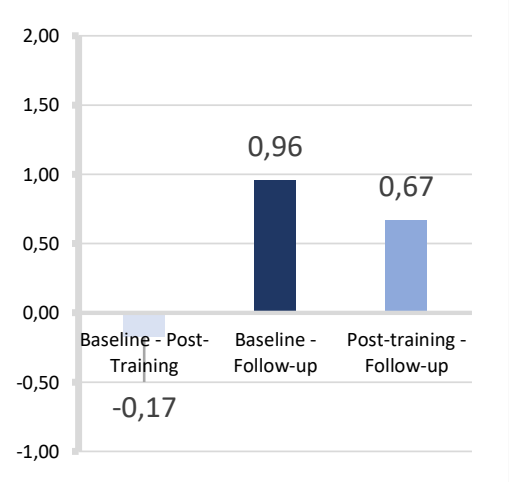

Notes: Effect sizes $(d)$ : $\quad$ small $(0.20-0.50)$, medium $(0.50-0.80)$, and $\square$ large $(0.80$ or higher) Positives ds indicate a decrease in pain/distress 
